\title{
Optimization of a bolometer detector for ITER based on Pt absorber on SiN membrane*
}

\author{
H Meister, ${ }^{\dagger}$ T Eich, N Endstrasser, L Giannone, M Kannamüller, A Kling, J Koll, T Trautmann, and ASDEX Upgrade Team \\ Max-Planck-Institut für Plasmaphysik, EURATOM Association, Boltzmannstr. 2, D-85748 Garching, Germany \\ P Detemple and S Schmitt \\ Institut für Mikrotechnik Mainz GmbH, Carl-Zeiss-Str. 18-20, D-55129 Mainz, Germany
}

\begin{abstract}
Any plasma diagnostic in ITER must be able able to operate at temperatures in excess of $200{ }^{\circ} \mathrm{C}$ and neutron loads corresponding to $0.1 \mathrm{dpa}$ over its lifetime. To achieve this aim for the bolometer diagnostic, a miniaturized metal resistor bolometer detector based on Pt absorbers galvanically deposited on SiN membranes is being developed. The first two generations of detectors featured up to $4.5 \mu \mathrm{m}$ thick absorbers. Results from laboratory tests are presented characterizing the dependence of their calibration constants under thermal loads up to $450{ }^{\circ} \mathrm{C}$. Several detectors have been tested in ASDEX Upgrade providing reliable data but also pointing out needs for further optimization. A laser trimming procedure has been implemented to reduce the mismatch in meander resistances below $1 \%$ for one detector and the thermal drifts from this mismatch.
\end{abstract}

\section{INTRODUCTION}

The total radiated power as well as the radiation emission profile on ITER will be determined by the bolometer diagnostic. A bolometer measures the plasma radiation over a wide spectral range (from soft- $\mathrm{X}$ to the infrared) by monitoring the temperature rise induced by deposition of photon energy in the absorber layer of the bolometer. The reference detector type chosen for ITER is the metal resistor bolometer ${ }^{1,2}$.

In operating fusion devices, bolometers mostly use a gold absorber on thin mica or kapton foils with gold meanders on their back side. Owing to the high neutron irradiation levels projected for ITER, these bolometers will fail because of the transmutation of $\mathrm{Au}$ to $\mathrm{Hg}$ and the embrittlement of the mica or kapton foils ${ }^{3}$. Materials reported to be more radiation resistant are $\mathrm{Pt}$ and $\mathrm{SiN}^{4,5}$. Thus, the detector development for ITER now focusses on Pt absorbers on SiN membranes.

The remainder of this paper is structured as follows. Section II reports on recently developed prototypes of bolometer detectors and their characterisation with respect to parameters relevant for operation. Section III gives examples of measurements performed in ASDEX Upgrade during the experimental campaign of 2009. Section IV reports on the development of a laser trimming procedure for the tailoring of the bolometer resistances. Finally, section V concludes with an outlook on further developments required.

\section{CHARACTERISATION OF PROTOTYPE SENSORS}

One of the most crucial developments for the final ITER bolometer diagnostic is the development of a radiation hard resistive bolometer foil with thick enough absorbers to reliably detect the total radiated power from the plasma. The thickness of the absorber has to match the attenuation length for the photon energies expected in ITER (up to $25 \mathrm{keV}$ in

${ }^{*}$ Contributed paper, published as part of the Proceedings of the 18th Topical Conference on High-Temperature Plasma Diagnostics, Wildwood, New Jersey, USA, May 2010. standard scenario). Therefore, it was proposed to develop a resistive bolometer detector with Pt-absorber of at least $12 \mu \mathrm{m}$ thickness on a $\mathrm{SiN}$ membrane which acts as isolator between the absorber on its one side and the metal resistor, made of meanders from $\mathrm{Pt}$, on its other side. The most efficient way to produce the required absorber thickness is to use galvanic deposition as one part of the procedure used to manufacture the detector chips out of a silicon wafer. During this procedure, first a silicon wafer is coated with a $\mathrm{Si}_{3} \mathrm{~N}_{4}$ layer $(1.5 \mu \mathrm{m})$ on which the Pt meanders ( $300 \mathrm{~nm}$ thick, $30 \mu \mathrm{m}$ wide) and $\mathrm{Pt}$ conductive paths $(600 \mathrm{~nm}$ thick, $300 \mu \mathrm{m}$ wide) are sputtered and evaporated. Then the $\mathrm{Si}$ is etched on the other side to open the windows for measurement and reference absorber until only the SiN remains as a free membrane. In the generated recess the Pt absorbers are deposited using an electroplating process. The layout of the prototype detectors matches that of the conventional ones ${ }^{1}$ to retain compatibility with existing hardware. So far, absorber thicknesses of $4.5 \pm 0.1 \mu \mathrm{m}$ have been achieved. Irradiation tests of a first sample ${ }^{6}$ demonstrated operation during the complete irradiation cycle.

To characterise the prototype detectors, a new test rig has been set up providing the capability to run the typical bolometer calibration procedure ${ }^{7}$ for up to three samples simultaneously at up to $450{ }^{\circ} \mathrm{C}$ in vacuum. The main parameters to be determined in dependence of the temperature are the values of meander resistances $R$, the cooling time constant $\tau$ and the normalised heat capacity $\kappa$ of the absorber as they enter the equations for deriving the radiational power.

\section{A. Meander resistances}

Heating the samples up to moderate temperatures of $200^{\circ} \mathrm{C}$ shows an absolute linear increase in resistance (FIG. 1, left). Typical temperature coefficients of resistivity (TCR) are in the order of $1.8 \mathrm{mK}^{-1}$. They are lower than the ones measured during the production process which range typically from 1.9$2.1 \mathrm{mK}^{-1}$. The reason is probably to be found in the differences of applying the elctrical contacts.

In ITER bolometers will have to operate at about $200^{\circ} \mathrm{C}$ or higher and might be exposed to temperatures in excess of 

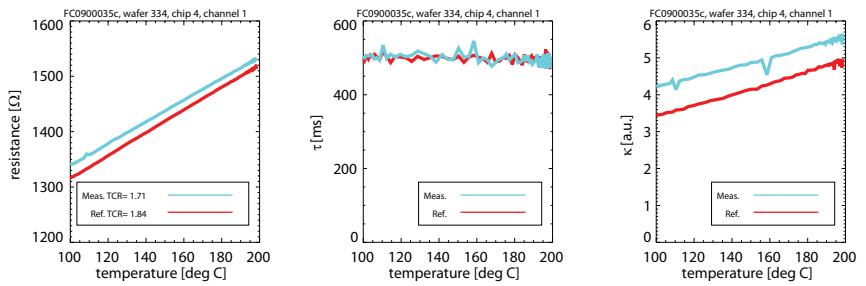

FIG. 1: Meander resistance (left), cooling time constant $\tau$ (middle) and normalized heat capacity $\kappa$ (right) for measurement (blue) and reference (red) absorber as functions of temperature.

$30{ }^{\circ} \mathrm{C}$ during unforeseen events or due to baking. Therefore, tests up to $450^{\circ} \mathrm{C}$ have been performed. In FIG. 2 it can be

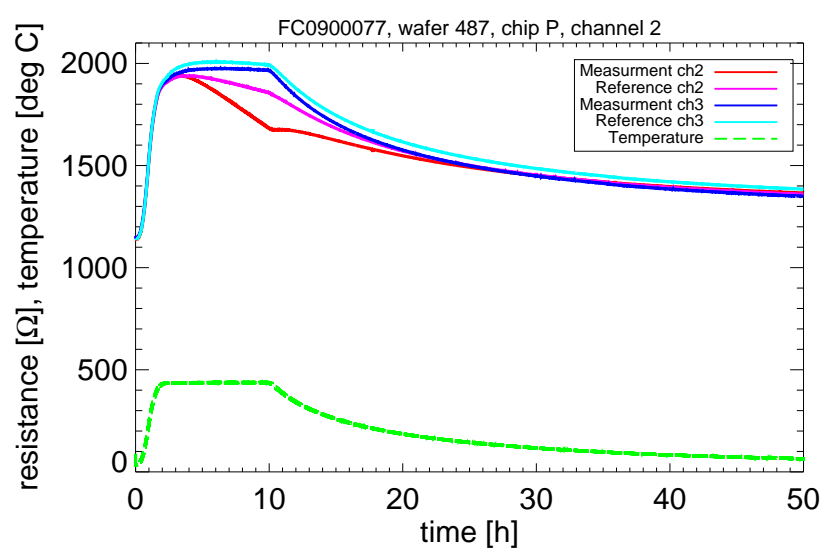

FIG. 2: Meander resistance of measurement and reference absorber for two channels of Chip P from wafer 487 as funtion of time. The time dependence of the temperature is given, too (green dashed).

seen that from about $430{ }^{\circ} \mathrm{C}$ onwards a drift in the meander resistance starts. In other cases the drift has been observed from $300^{\circ} \mathrm{C}$ onwards. This drift is not yet understood and behaves differently for each channel, ranging from almost no drift (channel 3 in FIG. 2) to very pronounced drifts (channel 2 in FIG. 2). Further tests have to clarify if the drifts are caused by problems when applying the elctrical contacts in the high-temperature environment of the test rig. Also it has to be investigated if the drift might reach a saturation if the samples are kept long enough at high temperatures.

\section{B. Cooling time constant}

The cooling time constants $\tau$ of the new samples are in the range of $450-550 \mathrm{~ms}$. Their variation with temperature has been observed to be both slightly positive or negative. In the case shown in the middle part of FIG. 1 it is almost constant. The original Au-detectors did show a pronounced dependence on temperature, but had cooling time constants in the order of $125 \mathrm{~ms}$. It has been proposed that the cooling time constant of the prototype bolometers is the result of the interplay of the temperature dependent specific heat and thermal conductivity of the low stress $\mathrm{SiN}$ membranes ${ }^{4}$.

\section{Heat capacity}

In the right part of FIG. 1 the normalized heat capacity $\kappa$ is plotted for one sample as a function of temperature. A clear linear dependence can be observed. In order to compare the absolute values to the ones of the original detectors, first the calibration procedure has to be adapted to the now longer cooling time constants.

\section{MEASUREMENTS IN ASDEX UPGRADE}

Several samples of the first batch of bolometer detectors with Pt-absorber (flow chart 0800076) have been mounted in bolometer heads of a camera in ASDEX Upgrade viewing the plasma from above the upper passive stabilising loop down into the divertor. They have been chosen such that corresponding lines-of-sight equipped with old Au-foils exist which have an almost identical viewing geometry. They have been acquiring data reliably during the whole experimental campaign of ASDEX Upgrade in 2009 and can be used for comparing the performance of the newly developed detectors with the existing ones.

FIG. 3 schows the line-integrated values of the plasma radiation measured with the old (light colour) and new (black) detectors plotted as function of the index of the line-of-sight. Both measurements show very good agreement to within $4 \%$.

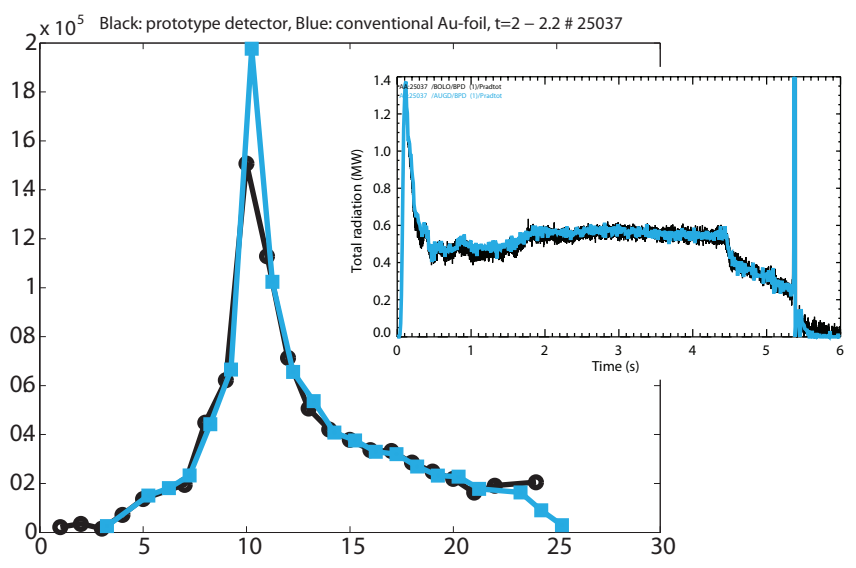

FIG. 3: Line-integrated radiation measurements acquired using the old (light colour) and new detectors (black), plotted against the index of the line-of-sight. The inset at the right hand side shows a comparison of the time trace of the total plasma radiation, evaluated from old and new detectors.

The differences visible are due to the slight offset in the geometry of the lines-of-sight.

The total plasma radiation has been evaluated for both, old and new detectors individually, too. They also agree very well, as can be seen in the inset in FIG. 3. However, the new detectors have a lower signal-to-noise ratio. The reason is that the capacitance between channels on one detector is up to a factor 
of 10 higher for the new detectors than for the old Au-foils due to the finite conductivity of the Si which constitutes the bulk material the new detectors are made of. Increasing the thickness of the $\mathrm{SiN}$ membrane from $1.5 \mu \mathrm{m}$ to $3 \mu \mathrm{m}$ reduces the capacitance between channels on one detector by a factor of 2, thus confirming the assumption that meanders on one side and $\mathrm{Si}$ on the other of the SiN membrane act as capacitor. In order to improve the signal-to-noise ratio, the reduction of the capacitance between channels has to be improved further.

\section{LASER TRIMMING OF MEANDER RESISTANCES}

Laser trimming is a widely used process in semi-conductor industry for the precise tailoring of resistancies of printed circuit paths. This method has now been adapted for tailoring the resistancies of the meanders of the bolometer resistors. Due to production tolerances during the deposition of the meanders the values of the resistancies for measurement and reference detector show variations in the order of $\pm 15 \Omega$. This leads to drifts of the measurement signal with changing ambient temperature, in particular when operating bolometers at elevated temperatures.

The laser trimming for bolometer meanders has been implemented using the laser trimmer LS-9520TD from Laser Systems GmbH. A pulsed $10 \mathrm{~W} \mathrm{Nd:YAG} \mathrm{laser} \mathrm{at} 1064 \mathrm{~nm}$ with a focus size of $12 \mu \mathrm{m}$ is used to cut bridges in the meander layout and thus increase its resistance in steps. A close-up of a trimming cut is shown in FIG. 4. During the trimming the
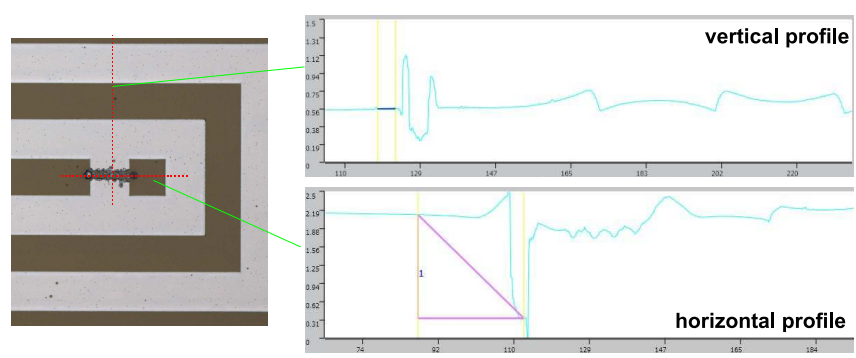

FIG. 4: Cut in a meander bridge generated by laser trimming (left). Profiles of the cut taken using a 3D scanning microscope (right).

laser melts the Pt and deposits it alongside and at the end of the cut as can be seen from the profiles of the cut shown on the right-hand side in FIG. 4. However, for a clean cut of the meander bridge the removal of some material of the SiN membrane cannot be avoided. Therefore, it is essential to optimise the power of the laser, the repetition frequency of the pulses and the cutting speed, i.e. the speed with which the laser is moved accross the sample surface. If Pt from the meander should flow through holes in the SiN membrane short-cuts might be produced. The best results for current samples have been achieved at $85 \%$ laser power, $10 \mathrm{kHz}$ pulse frequency and $45 \mathrm{~mm} / \mathrm{s}$ cutting speed.

Each cut of the bridges in the current mask layout increases the meander resistance by about $\Delta R_{\text {cut }}=2.5 \Omega$. The theoretically achievable accuracy for matching the resistances of one channel is $0.2 \%$ for typical meander resistancies of $1200 \Omega$. However, tests result in accuracies of typically $0.8 \%$ and the average change in resistance per cut is below $2 \Omega$.The reason is that, depending on the location on the sample, not all material is cleanly removed for all cuts thus resulting in a less pronounced increase of resistance. Better results will be feasible by optimising the software of the computer controlled trimming process. Most probably, the vertical position of the focus of the laser is not yet defined correctly. Additionally, more sophisticated trimming features in the mask layout of the meanders could be foreseen which enable a high total change in resistance and variable resistance changes for the final trimming cuts.

The effort to reduce the bridge imbalance as much as possible will pay off even for the case of irradiated samples. During irradiation the resistances of meanders change but those belonging to one channel should change in a similar way as it has to be ascertained that they are exposed to the same irradiation level for a reliable signal evaluation. Thus, the closer the resistances are matched at the start of operation the less differences can be expected after the irradiation. This, of course, has to be proven experimentally.

\section{CONCLUSION}

The results presented show that the development of a new prototype bolometer detector is well underway. However, they also demonstrate that further improvements for operation in ITER are still necessary. Obviously, the absorber thickness has to be increased . Furthermore, the hysterisis of the meander resistance at temperatures when heated above $300^{\circ} \mathrm{C}$ has to be understood. Also, the capacitance between channels on one detector have to be reduced for enhancing the signal-tonoise ratio for high bandwidth operation.

To fully optimize the prototype bolometer detector for operation in ITER, closely matched meander resistance and calibration parameters ( $\tau$ and $\kappa$ ) are required in order to minimize the thermal drifts in the measurement due to changes in ambient temperature during long pulses. The first results with laser trimming of the meander resistances indicate that matching of the meander resistances is possible and further optimization of the laser and the trimming procedure will improve the matching to the theoretically achievable level. $\dagger$ Electronic address: meister@ipp.mpg . de

1 K. F. Mast, J. C. Vallet, C. Andelfinger, P. Betzler, H. Kraus, and G. Schramm, rsi 62, 744 (1991).
2 H. Meister, L. Giannone, L. D. Horton, G. Raupp, W. Zeidner, G. Grunda, S. Kalvin, U. Fischer, A. Serikov, S. Stickel, et al., Review of Scientific Instruments 79 (2008). 
3 T. Nishitani, T. Shikama, R. Reichle, E. R. Hodgson, E. Ishitsuka, S. Kasai, and S. Yamamoto, fed 63-64, 437 (2002).

4 L. Giannone, D. Queen, F. Hellman, and J. C. Fuchs, ppcf 47, 2123 (2005).

5 M. Gonzalez and E. R. Hodgson, fed 82, 1277 (2007).

6 A. Gusarov, S. Huysmans, L. Giannone, and H. Meister, private communication, to be published in Fusion Engineering and Design (2010).

7 L. Giannone, K. F. Mast, M. Schubert, N. Team, E. Team, and W. Team, rsi 73, 3205 (2002). 